\title{
Formation and structural characterization of Ni nanoparticles embedded in $\mathrm{SiO}_{2}$
}

\author{
D. J. Sprouster, ${ }^{1, a)}$ R. Giulian, ${ }^{1}$ L. L. Araujo, ${ }^{1}$ P. Kluth, ${ }^{1}$ B. Johannessen, ${ }^{2}$ N. Kirby, ${ }^{2}$ \\ and M. C. Ridgway ${ }^{1}$ \\ ${ }^{1}$ Department of Electronic Materials Engineering, Research School of Physics and Engineering, \\ Australian National University, Canberra, Australian Capital Territory 0200, Australia \\ ${ }^{2}$ Australian Synchrotron, Clayton, Victoria 3168, Australia
}

(Received 23 December 2010; accepted 26 April 2011; published online 7 June 2011)

\begin{abstract}
Face-centered cubic Ni nanoparticles were formed in $\mathrm{SiO}_{2}$ by ion implantation and thermal annealing. Small-angle x-ray scattering in conjunction with transmission electron microscopy was used to determine the nanoparticle size as a function of annealing temperature, whereas the local atomic structure was measured with x-ray absorption spectroscopy. The influence of finite-size effects on the nanoparticle structural properties was readily apparent and included a decrease in coordination number and bond length and an increase in structural disorder for decreasing nanoparticle size. Such results are consistent with the non-negligible surface-to-volume ratio characteristic of nanoparticles. In addition, temperature-dependent $\mathrm{x}$-ray absorption spectroscopy measurements showed the mean vibrational frequency (as obtained from the Einstein temperature) decreased with decreasing nanoparticle size. This reduction was attributed to the greater influence of the loosely bound, under-coordinated surface atoms prevailing over the effects of capillary pressure, the former enhancing the low frequency modes of the vibrational density of states. (C) 2011 American Institute of Physics. [doi:10.1063/1.3594751]
\end{abstract}

\section{INTRODUCTION}

Nanoparticles (NPs) are of growing interest due to their novel magnetic and optical properties that can deviate considerably from those of bulk material. Such differences stem from two major effects, namely: (i) a large relative fraction of surface atoms compared to the NP core (or large surface-to-volume ratio) and (ii) carrier confinement in nanometer-sized particles. The large surface-to-volume ratio for small NPs results in a depression in the melting temperature for metallic $\mathrm{NPs}^{1,2}$ and significant structural perturbations including reduced coordination numbers and an increase in structural disorder $^{3}$ compared to the bulk. Electron confinement in metallic NPs yields the unique optical and magnetic properties, including intense light absorption in the visible region, ${ }^{4}$ enhanced nonlinear optical properties, ${ }^{5}$ reduced magnetic anisotropy barriers, and enhanced magnetic moments compared to the bulk. ${ }^{6}$

Ion beam synthesis (IBS) is a novel NP fabrication method with many advantages over other formation techniques and, as a consequence, has been widely used for the fabrication of both metallic and semiconductor NPs in various host matrices. ${ }^{7,8}$ The versatility of IBS stems from the ability to: (i) introduce effectively any impurity into a desired host matrix without solid solubility limitations and (ii) control of the implanted ion concentration and depth within the matrix by manipulation of the implantation fluence and energy, respectively. Ni NPs formed by IBS are of particular interest as the optical and magnetic properties are highly dependent on the host matrix, ${ }^{9-11}$ post-implantation annealing atmosphere, ${ }^{12}$ and size. ${ }^{13}$ However, very few studies have

${ }^{\text {a)} E l e c t r o n i c ~ m a i l: ~ d j s 109 @ p h y s i c s . a n u . e d u . a u . ~}$ discussed in detail the effects of post-implantation annealing on the structure and size of Ni NPs formed by IBS.

The vibrational properties of NPs are also of fundamental and technological importance, as the vibrational density of states (VDOS) is key to the understanding of the thermodynamic properties including the heat capacity, vibrational entropy, superconductivity transition temperature, and melting temperature. There have been numerous theoretical studies of the influence of finite-size effects on the VDOS of small Ni NPs (see, e.g., Refs. 14-16), although validation via experimental studies is scant.

In the present article, we present a size-dependent structural characterization study of Ni NPs formed by IBS. Smallangle x-ray scattering (SAXS) is used in conjunction with transmission electron microscopy (TEM) to determine the NP size, whereas $x$-ray absorption spectroscopy is used to probe the NP structural and vibrational properties. The manuscript is organized as follows: In Sec II we present the experimental procedures for the formation and characterization of the Ni NPs, in Sec. III the experimental results and discussion of the key findings, including the size-dependent structural and vibrational properties of Ni NPs are presented, and, finally, in Sec. IV we summarize our main conclusions.

\section{EXPERIMENT}

$\mathrm{Ni}$ ions of $1 \mathrm{MeV}$ were implanted at liquid- $\mathrm{N}_{2}$ temperature and to a fluence of $3 \times 10^{17} \mathrm{~cm}^{-2}$ into $2-\mu$ m-thick $\mathrm{SiO}_{2}$ layers thermally grown on $\mathrm{Si}(100)$ substrates. The peak $\mathrm{Ni}$ concentration, calculated using TRIM, ${ }^{17}$ was 9 at. $\%$ at a depth of $1 \mu \mathrm{m}$. Samples were then annealed at $800-1100{ }^{\circ} \mathrm{C}$ in forming gas $\left(5 \% \mathrm{H}_{2}+95 \% \mathrm{~N}_{2}\right)$ for $0.5-1 \mathrm{~h}$ to promote metal precipitation and NP growth. 
For the synchrotron measurements, a unique sample preparation method ${ }^{18}$ was used to isolate the thin $\mathrm{SiO}_{2}$ layer containing the Ni NPs: The Si substrate was removed through mechanical grinding and selective wet chemical etching with $\mathrm{KOH}$, then multiple $\mathrm{SiO}_{2}$ layers were stacked together between two Kapton windows. As a consequence, considerable NP material was concentrated within the sample holder, scattering from the Si substrate was eliminated and high-resolution measurements were enabled. Crystalline $\mathrm{Ni}$ and $\mathrm{NiO}$ standards were prepared by sputtering a $\mathrm{Ni}$ film of $200 \mathrm{~nm}$ onto a $\mathrm{SiO}_{2} / \mathrm{Si}$ wafer at room temperature (for the $\mathrm{Ni}$ standard) and at $400{ }^{\circ} \mathrm{C}$ in an $\mathrm{O}$ atmosphere (for the $\mathrm{NiO}$ standard). A $50 \mathrm{~nm}$ capping layer of $\mathrm{SiO}_{2}$ was then deposited on both standards to prevent further oxidation. X-ray diffraction confirmed the crystalline phase of both standards.

Transmission SAXS measurements were performed at beamline 13-ID of the Australian Synchrotron using 11.3 $\mathrm{keV}$ x rays. The scattering intensity $I(Q)$ was collected for 5 $\mathrm{s}$ at a camera length of $1871 \mathrm{~mm}$. A scattering pattern from an unimplanted $\mathrm{SiO}_{2}$ sample was subtracted from all NP spectra. Size distributions were determined using the nonlinear least square fitting procedures in the IGOR-Pro-based software package from NIST. ${ }^{19}$ Spherical particles were assumed, consistent with TEM analysis.

TEM measurements were used to determine the NP shape and size. Electron transparency was achieved with conventional mechanical thinning, polishing, and dimpling followed by ion milling. TEM images and diffraction patterns were recorded with a Phillips CM300 microscope operating at $300 \mathrm{kV}$.

Fluorescence-mode $\mathrm{x}$-ray absorption near edge structure (XANES) and extended x-ray absorption fine structure (EXAFS) measurements were performed at beamline 20-B of the Photon Factory. Samples were measured at the Ni $K$ edge $(8.333 \mathrm{keV})$ between temperatures of $15-300 \mathrm{~K}$. Spectra were recorded using a $6 \times 6$-pixel-array $\mathrm{Ge}$ detector with the Si (111) monochromator detuned by $50 \%$ for harmonic rejection. Data were collected to a photoelectron wave number $(k)$ value of $16 \AA^{-1}$. For energy calibration, a $\mathrm{Ni}$ face-centered-cubic (fcc) reference foil was simultaneously measured in transmission mode. Background subtraction, spectra alignment, and normalization of the EXAFS data were performed with ATHENA. ${ }^{20}$ Isolated EXAFS spectra were Fourier-transformed (FT) over a $k$-range of 3.3-15.7 $\AA^{-1}$ with an adaptive Hanning window and back FT over a nonphase-corrected radial distance range of either 1.6-2.7 (for the Ni standard and annealed samples) or 1.1-3.1 $\AA$ (for the $\mathrm{NiO}$ standard and as-implanted sample) to isolate the nearest neighbor(s) of interest. Structural parameters were determined with ARTEMIS ${ }^{20}$ utilizing the IFEFFIT ${ }^{21}$ code with theoretical scattering amplitudes and phase shifts calculated $a b$ initio with FEFF8. ${ }^{22}$ The amplitude reduction factors $\left(S_{2}^{0}=0.87 \pm 0.03\right.$ and $\left.0.91 \pm 0.06\right)$ and energy shift parameters $\left(E_{0}=2.04 \pm 0.23\right.$ and $\left.2.46 \pm 0.50 \mathrm{eV}\right)$ were determined for the $\mathrm{Ni}$ and $\mathrm{NiO}$ standards and then fixed for the subsequent fitting of all NP spectra. The coordination numbers $(\mathrm{CN})$ for the bulk standards were fixed at the theoretical values and floated for the NP samples. The bond length $(R)$, Debye-Waller factor $\left(\sigma^{2}\right)$, and third cumulant $\left(C_{3}\right)$ for each sample were allowed to vary during the fitting. For the measurement-temperature-dependent EXAFS analysis, the evolution of the first nearest-neighbor structural parameters was determined by analyzing each data set over the 15-300 $\mathrm{K}$ temperature range individually with the $\mathrm{CN}$ fixed to the values determined at $15 \mathrm{~K}$. The $R, \sigma^{2}$, and $C_{3}$ were allowed to vary freely and the temperature dependence of the $\sigma^{2}$ was fit with an anharmonic correlated Einstein model [the forthcoming Eq. (2)] to determine the static and temperature-dependent contributions to the total disorder.

\section{RESULTS AND DISCUSSION}

\section{A. NP size as a function of annealing temperature}

The background subtracted and fitted SAXS spectra are shown in Fig. 1(a) as a function of the scattering vector $Q$ for different annealing conditions. The pronounced contribution to the scattering intensity in all samples clearly reflects the high electron density of the Ni NPs compared to the $\mathrm{SiO}_{2}$ matrix. The superimposed lines show the corresponding fits with a Schulz distribution, ${ }^{23}$ which are in excellent agreement with the experimental data. The reconstructed normalized size distributions (volume weighted) are shown in
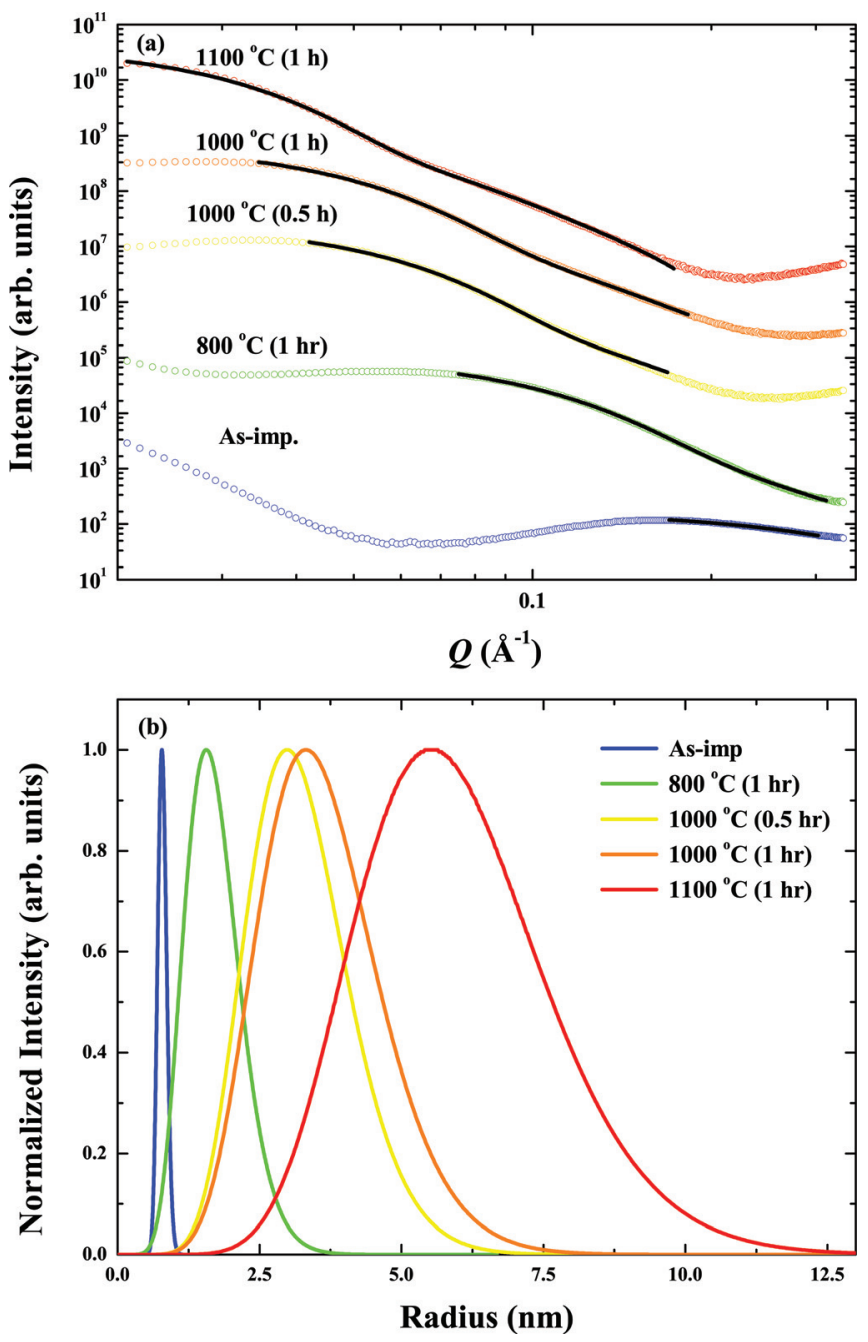

FIG. 1. (Color online) (a) Background-subtracted SAXS intensities (fitted region shown in black with spectra offset for comparison) and (b) normalized, volume-weighted NP size distributions deduced from SAXS. 
TABLE I. Refined EXAFS fitting parameters and SAXS size distributions. ${ }^{a}$

\begin{tabular}{|c|c|c|c|c|c|}
\hline Sample ${ }^{\mathrm{b}}$ & $R(\AA)$ & $\sigma^{2}\left(10^{-3} \AA^{2}\right)$ & $\mathrm{CN}$ (atoms) & $C_{3}\left(10^{-5} \AA^{3}\right)$ & Radius (nm) \\
\hline Bulk Ni foil & $2.474(0.001)$ & $2.76(0.07)$ & 12 (fixed) & $-1.0(0.7)$ & \\
\hline $1100^{\circ} \mathrm{C}$ & $2.473(0.001)$ & $2.78(0.15)$ & $11.5(0.4)$ & $-3.7(1.2)$ & $6.0(1.7)$ \\
\hline $1000^{\circ} \mathrm{C}$ & $2.471(0.001)$ & $2.97(0.05)$ & $11.1(0.3)$ & $-3.4(0.7)$ & $3.7(1.1)$ \\
\hline $1000^{\circ} \mathrm{C}^{\mathrm{c}}$ & $2.470(0.001)$ & $3.09(0.07)$ & $10.5(0.3)$ & $-3.5(0.7)$ & $3.2(0.9)$ \\
\hline $800^{\circ} \mathrm{C}$ & $2.467(0.001)$ & $3.31(0.07)$ & $9.3(0.2)$ & $-3.1(1.2)$ & $1.6(0.5)$ \\
\hline Bulk $\mathrm{NiO}(\mathrm{Ni}-\mathrm{O})$ & $2.061(0.005)$ & $4.08(0.61)$ & 6 (fixed) & $-15.4(14.1)$ & \\
\hline Bulk NiO (Ni-Ni) & $2.940(0.002)$ & $3.28(0.21)$ & 12 (fixed) & $-0.5(2.8)$ & \\
\hline As-impl. (Ni-O) & $1.869(0.018)$ & $6.13(2.82)$ & $0.7(0.3)$ & $\mathrm{nm}$ & $0.9(0.1)$ \\
\hline As-impl. $(\mathrm{Ni}-\mathrm{Ni})$ & $2.454(0.003)$ & $8.2(0.43)$ & $3.9(0.7)$ & $\mathrm{nm}$ & \\
\hline
\end{tabular}

${ }^{\mathrm{a}} R, \sigma^{2}, \mathrm{CN}$, and $C_{3}$ are the bond-length, Debye-Waller factor, coordination number, and third cumulant, respectively.

${ }^{\mathrm{b}}$ Samples were annealed for $1 \mathrm{~h}$.

${ }^{\mathrm{c}}$ This sample was annealed for $0.5 \mathrm{~h}$.

Fig. 1(b) and the average NP radius for all samples are given in Table I. Bright-field TEM images of the Ni NPs are shown in Fig. 2 and demonstrate that all NPs are spherical with no evidence of faceting at this magnification. Electron diffraction patterns (not shown) for all annealed samples exhibited well-defined Debye-Scherrer rings typical of randomly oriented fcc NPs. Both Figs. 1 and 2 show that the average NP size and the distribution of NP sizes increases with increasing annealing temperature.

Figure 3 shows an Arrhenius plot of the mean NP radius ( $r$ ) for all samples. Two distinct temperature dependencies are observed and both were fitted with the standard Arrhenius equation:

$$
\ln (r)=\ln (A)-E_{a}\left(1 / k_{\beta} T\right)
$$

where $A$ is a pre-exponential factor, $E_{a}$ is the activation energy, $k_{\beta}$ is the Boltzmann constant, and $T$ is the annealing
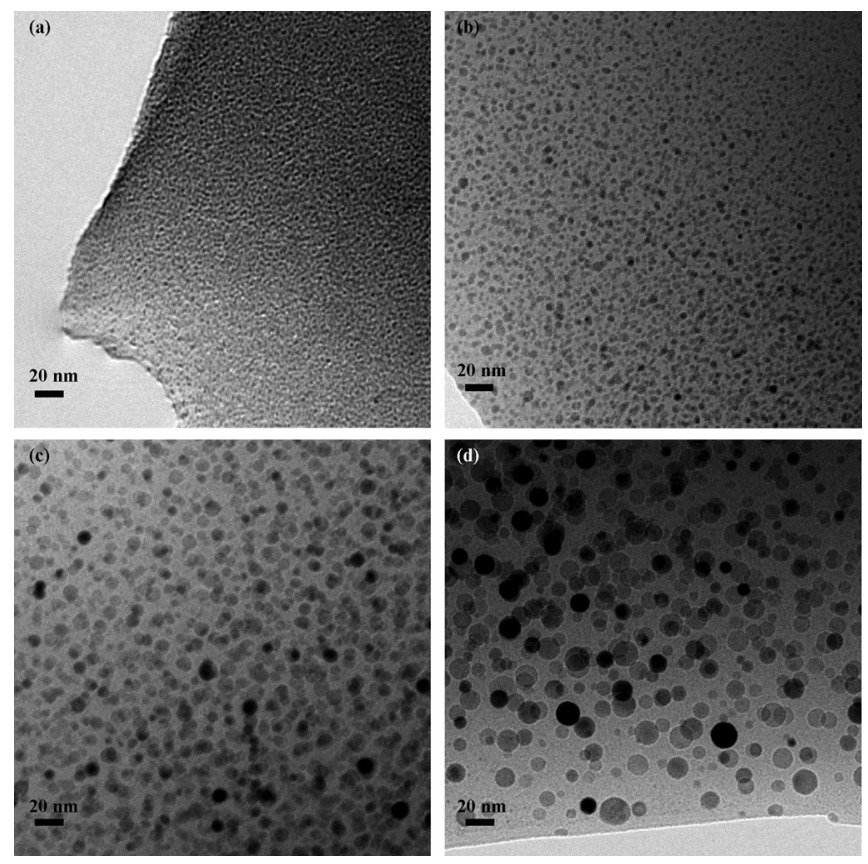

FIG. 2. TEM micrographs of (a) as-implanted and annealed (1 h) samples (b) $800{ }^{\circ} \mathrm{C}$, (c) $1000{ }^{\circ} \mathrm{C}$, and (d) $1100{ }^{\circ} \mathrm{C}$. temperature. For the low temperature region, an activation energy of $0.02 \pm 0.005 \mathrm{eV}$ was calculated (dashed line in Fig. 3). This practically athermal value was attributed to irradiation-enhanced diffusion at temperatures where thermally induced diffusion is minimal or negligible. ${ }^{24,25}$ At elevated temperatures $\left(\geq 800{ }^{\circ} \mathrm{C}\right)$, the activation energy was $0.53 \pm 0.09 \mathrm{eV}$ (solid line in Fig. 3) and consistent with increased $\mathrm{Ni}$ diffusivity. Similar activation energies have previously been reported for $\mathrm{Co}(0.59 \mathrm{eV})^{26}$ and $\mathrm{Cu}(0.60$ $\mathrm{eV})^{27}$ NPs formed under comparable implant conditions and indicates that the growth kinetics of these three metallic NPs formed by IBS are indeed similar.

\section{B. Size-dependent atomic structure}

Ni $K$-edge XANES spectra are shown in Fig. 4 for the bulk standards and the NP samples as a function of annealing temperature. XANES at the $K$-edge involves the excitation of a $1 s$ photoelectron with features above the absorption edge resulting from multiple-scattering resonances. The features of the XANES spectra for the fcc Ni and rock salt $\mathrm{NiO}$ standards are consistent with those previously reported. ${ }^{28}$

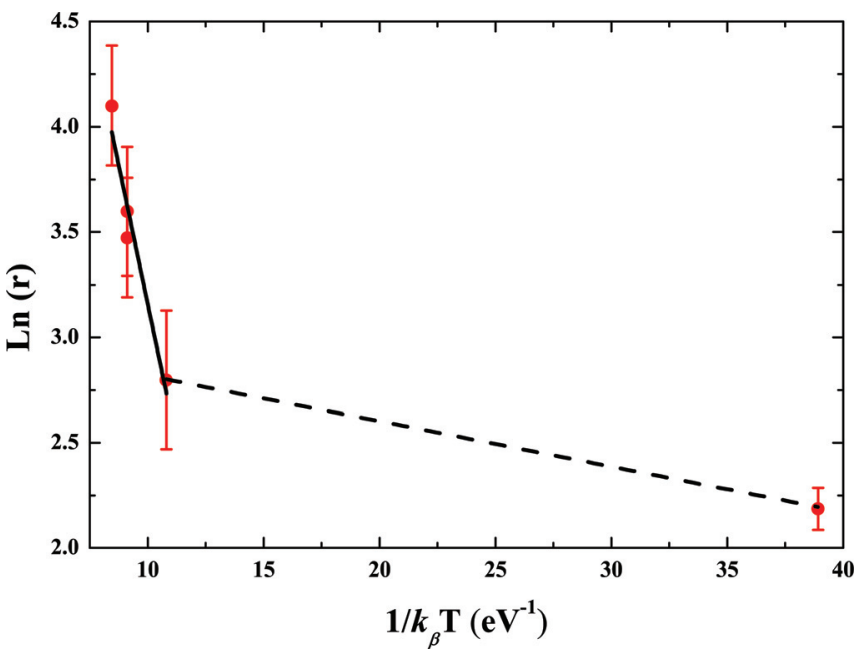

FIG. 3. (Color online) Arrhenius plot of the mean NP radius $(r)$ as a function of inverse $k_{\beta} T$ where $T$ is the annealing temperature and $k_{\beta}$ is the Boltzmann constant. The lines are linear fits to the experimental data with Eq. (1). 


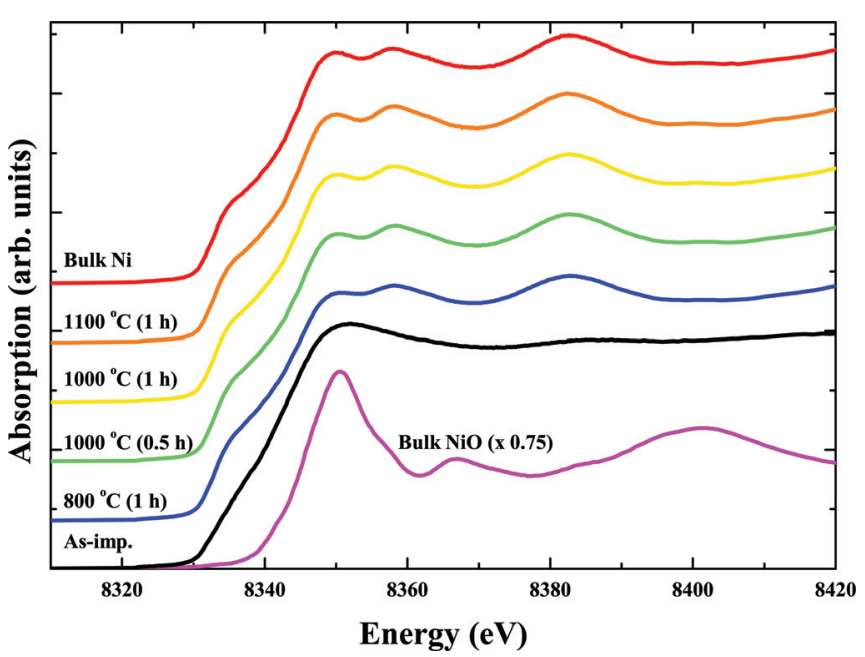

FIG. 4. (Color online) XANES spectra at the Ni $K$-edge for bulk standards and NP samples.

For the latter, the shift to higher energy is the result of the increase in $\mathrm{Ni}$ valence. XANES analysis reveals that the annealed Ni NPs formed by IBS have the metallic fcc structure of bulk $\mathrm{Ni}$, as consistent with electron diffraction results. Ni NPs formed by IBS display a higher degree of structural order compared to chemically synthesized NPs, which have a complex, disordered structure. ${ }^{29}$ The origin of the latter is still under investigation, but could potentially result from chemical impurities introduced during reduction. The lack of impurities in NPs formed by IBS again demonstrates the usefulness of this method for the controlled growth of metallic NPs.

The XANES of the as-implanted sample differs considerably when compared to the two bulk standards ( $\mathrm{Ni}$ and $\mathrm{NiO}$ ) and is essentially featureless. SAXS and TEM measurements (Figs. 1 and 2, respectively) show that very small NPs are present even in the as-formed state. The XANES and EXAFS results (detailed in the following) indicate that the as-implanted NPs consist of metallic $\mathrm{Ni}$, but are in a highly disordered state, similar to those in Ref. 29.

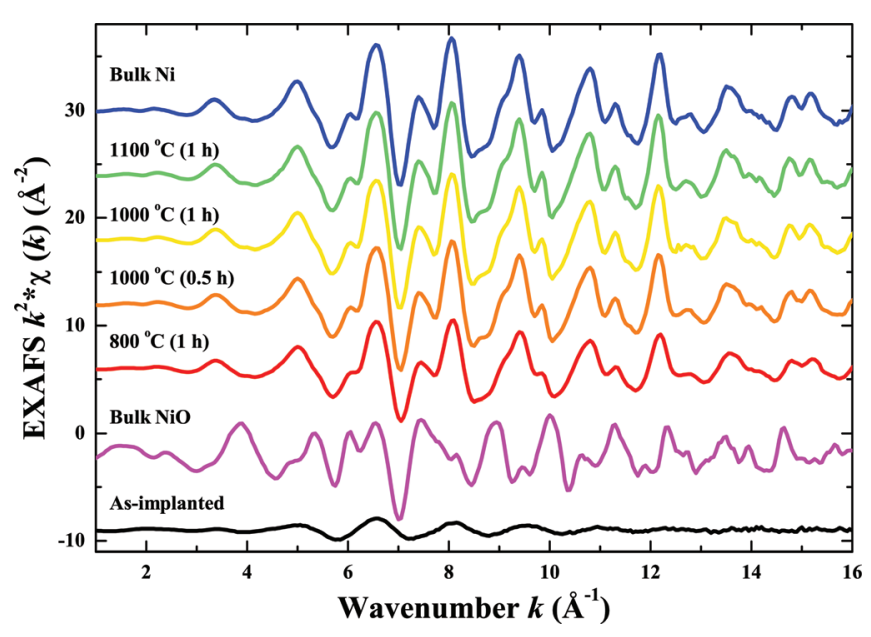

FIG. 5. (Color online) $k^{2}$-weighted EXAFS spectra for bulk Ni and all NP samples.

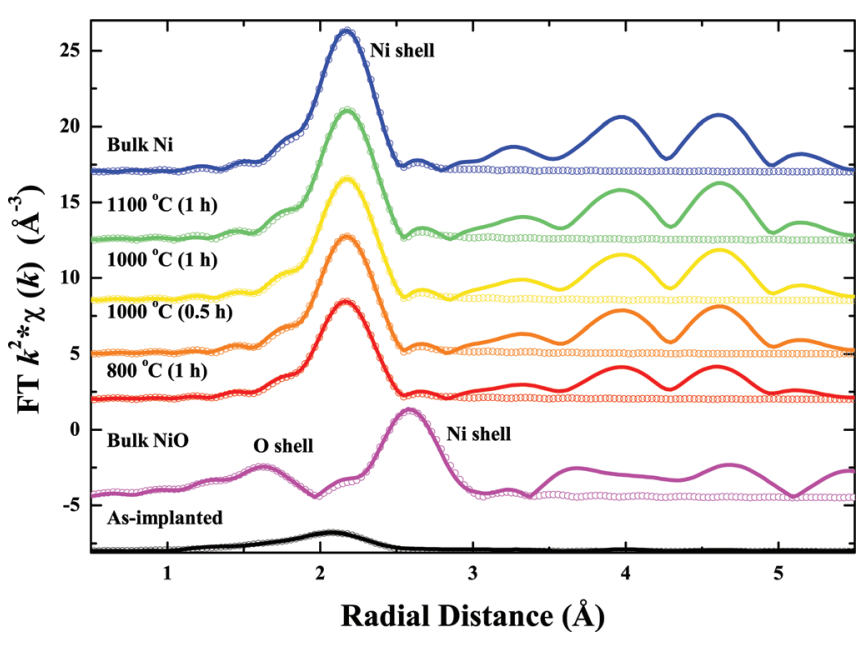

FIG. 6. (Color online) Phase-corrected FT EXAFS spectra for bulk Ni and all NP samples (fits to the first NN shell shown as symbols).

Figure 5 shows the $k^{2}$-weighted normalized Ni $K$-edge EXAFS oscillations $\chi$ as a function of wave number $k$ for the bulk standards and NP samples. The EXAFS amplitude increases with increasing annealing temperature, consistent with NP growth. Figure 6 shows the corresponding nonphase-corrected FT spectra. The amplitude of the FT spectra is highest for the Ni standard and decreases for decreasing annealing temperature or, equivalently, decreasing NP size. The peak between 2 and $3 \AA$ results from single-scattering photoelectron events between the absorbing atom and the first nearest-neighbor shell of atoms. The signal at higher distances (between 3 and $6.5 \AA$ ) is the superposition of both single-scattering photoelectron paths from the more distant shells and the contributions from multiple scattering paths. The decrease in the nearest-neighbor peak amplitudes and slight broadening with decreasing NP size reflects a decrease in the average $\mathrm{CN}$ and an increase in the disorder $\left(\sigma^{2}\right)$, both attributable to the increase in surface-to-volume ratio for small NPs. The superimposed dots in Fig. 6 are the corresponding fits to the first nearest-neighbor shell and structural parameters are given in Table I.

The Ni-Ni analysis for the bulk standard and NP samples summarized in Table I readily reveals size-dependent trends in the structural parameters. These quantitatively confirm the results discussed previously and include a reduced $\mathrm{CN}$, a contraction in $R$, an increase in $\sigma^{2}$, and a negative $C_{3}$ for decreasing NP size. Similar trends have been reported for other elemental metal NPs formed by IBS. ${ }^{25,26,30,31}$ As the NP size decreases, the relative fraction of under-coordinated

TABLE II. Einstein temperature $\left(\Theta_{E}\right)$ and structural disorder $\left(\sigma_{S}^{2}\right)$ obtained from temperature-dependent EXAFS analysis.

\begin{tabular}{lcc}
\hline \hline Sample & $\Theta_{E}(\mathrm{~K})$ & $\sigma_{S}^{2}\left(10^{-3} \AA^{2}\right)$ \\
\hline Bulk Ni & $299.5(1.5)$ & $0.12(0.03)$ \\
$3.7(1.1) \mathrm{nm}$ & $297.1(1.4)$ & $0.12(0.03)$ \\
$3.2(0.9) \mathrm{nm}$ & $296.4(1.5)$ & $0.17(0.03)$ \\
$1.6(0.5) \mathrm{nm}$ & $292.3(1.3)$ & $0.52(0.03)$ \\
\hline \hline
\end{tabular}


surface atoms increases leading to a decrease in the average $\mathrm{CN}$. The increase in $\sigma^{2}$ with decreasing NP size is also attributable to the increasing surface-to-volume ratio as disordered surface atoms reconstruct to accommodate material and bonding differences across the NP-matrix interface. ${ }^{32}$ The negative $C_{3}$ measured for all NP samples indicates that the bond-length distribution is skewed toward shorter distances and is attributed to the contracted bonds associated with the surface/interfacial atoms.

Table I demonstrates there is a mean bond-length contraction as the NP size decreases. This contraction results from capillary pressure induced by surface tension, where the former is proportional to inverse NP size. ${ }^{14}$ Using a simple liquid-drop model, ${ }^{33}$ the surface tension $f$ is calculable from $\Delta R=-(4 / 3) K R_{b} f(1 / D)$, where $\Delta R$ is the relative contraction in $R, K$ is the bulk compressibility, and $R_{b}$ is the bulk bond length. Using $K$ and $R_{b}$ for bulk Ni yields a surface tension of $1.9 \pm 0.3 \mathrm{~J} / \mathrm{m}^{2}$, identical (within error) to that predicted by Meyer et al. ${ }^{14}$ although also comparable to the bulk value of $1.7 \mathrm{~J} / \mathrm{m}^{2} .{ }^{34}$ Other elemental metal NPs embedded in $\mathrm{SiO}_{2}$ exhibit a similar trend [Pt (Ref. 25), Au (Ref. 31), and $\mathrm{Cu}$ (Ref. 30) NPs have surface tensions of 1.7, 3.6, and $7.0 \mathrm{~J} / \mathrm{m}^{2}$ respectively].

A bond-length contraction was also observed in Ni NPs deposited onto polymer films, ${ }^{35}$ although such NPs were appreciably smaller $(0.4-0.6 \mathrm{~nm}$ in radius) than those studied here and the contraction was attributed to changes in their electronic configuration. Given the similarity in the XANES spectra for all the NPs, we conclude changes in electronic configuration do not yield the bond-length contraction observed herein.

The EXAFS and FT spectra for the as-implanted sample shown in Figs. 5 and 6, respectively, both exhibit significantly less structure compared to those annealed at higher temperatures. This difference is attributed to multiple $\mathrm{Ni}$ environments including metallic Ni atoms in very small NPs and a small fraction of oxidized $\mathrm{Ni}$ atoms dissolved in the matrix in a disordered, noncrystalline arrangement. The structural parameters for the $\mathrm{Ni}-\mathrm{Ni}$ and $\mathrm{Ni}-\mathrm{O}$ shells are given in Table I. The increase in $\sigma^{2}$ for the $\mathrm{Ni}-\mathrm{O}$ shell in the as-implanted sample indicates that, as expected, the small fraction of oxidized $\mathrm{Ni}$ is indeed in a more disordered form than in the crystalline $\mathrm{NiO}$ standard. The significant reduction in the Ni-Ni bond length relative to the annealed samples is attributed to the very small size of the Ni NPs in the as-formed state. Further, the Ni-Ni EXAFS results demonstrate that the as-implanted NPs are not in a purely amorphous phase given that the trends in the structural properties are not consistent with previous work. ${ }^{36}$

\section{Size-dependent vibrational properties}

The vibrational properties of Ni NPs were probed with temperature-dependent EXAFS measurements. The FT spectra for bulk Ni and Ni NPs of $1.6 \mathrm{~nm}$ radii are shown in Fig. 7 as a function of the measurement temperature. The decrease in amplitude with an increase in temperature results from the increase in thermal disorder. The experimental $\sigma^{2}$ values determined from the EXAFS data are plotted as a
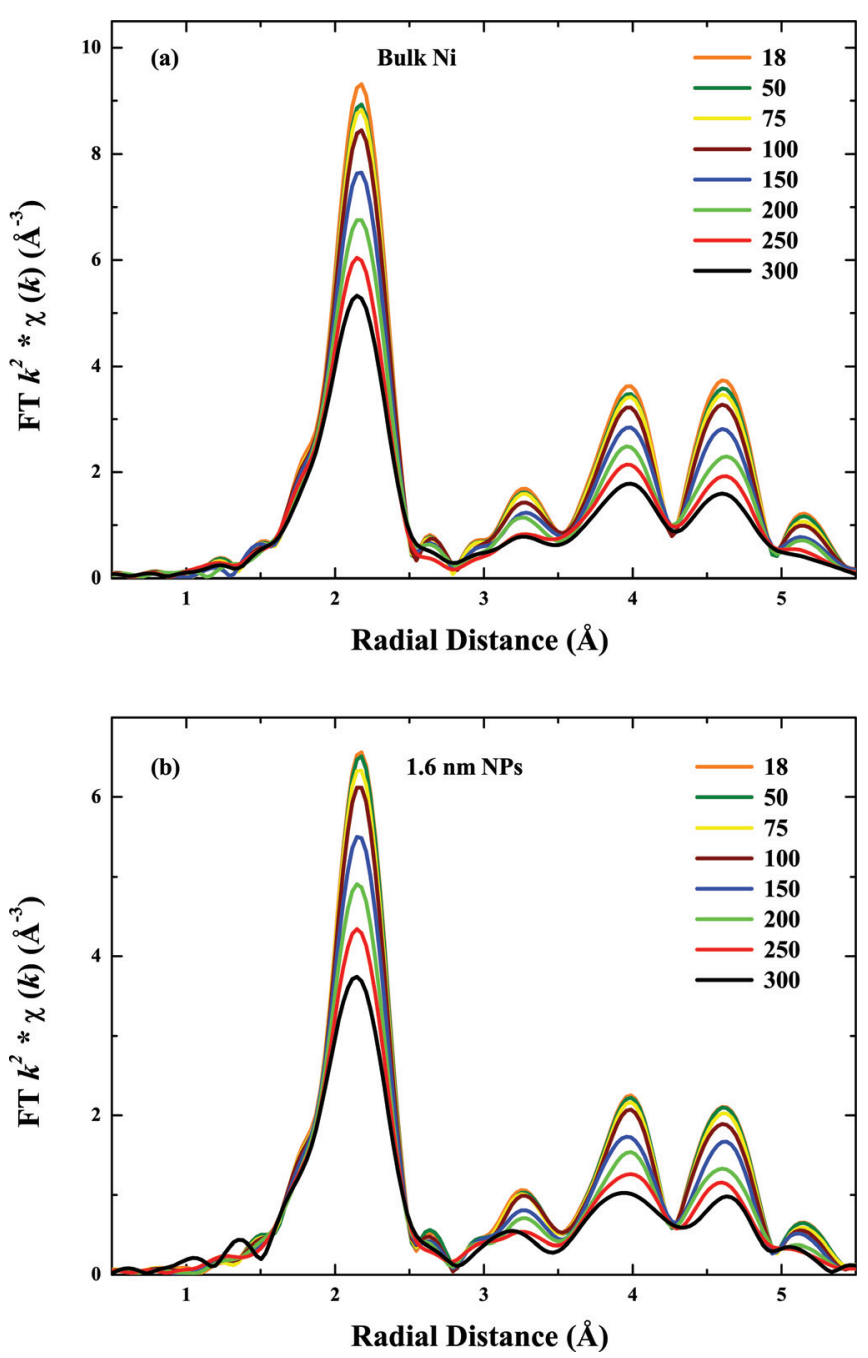

FIG. 7. (Color online) FT EXAFS spectra for (a) bulk Ni and (b) $1.6 \mathrm{~nm} \mathrm{Ni}$ NPs as a function of measurement temperature. Temperatures are given in degrees Kelvin.

function of measurement temperature in Fig. 8. The $\sigma^{2}$ is vertically offset for the NPs due to enhanced structural disorder. The $\sigma^{2}$ increases with temperature more rapidly for the NP samples compared to bulk Ni yielding a lower Einstein

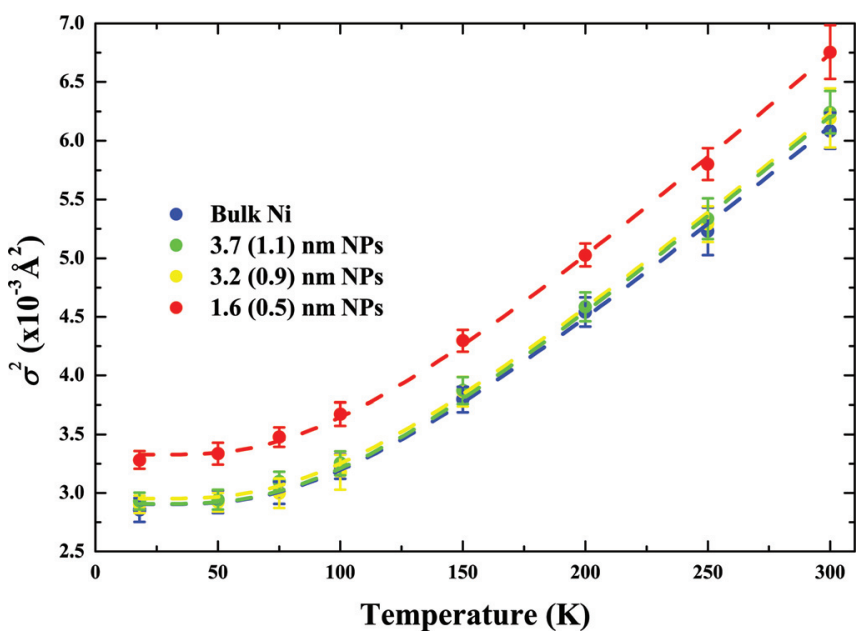

FIG. 8. (Color online) Thermal evolution of $\sigma^{2}$ for bulk Ni and NP samples. Lines are fits with Eq. (2). 
temperature and indicating that the NPs have looser (less stiff) bonding relative to bulk material. The corresponding best fits with the correlated Einstein model $l^{37,38}$ is given by

$$
\sigma^{2}=\frac{\hbar^{2}}{2 \mu k_{B} \Theta_{E}} \cdot \operatorname{coth}\left(\frac{\Theta_{E}}{2 T}\right)+\sigma_{\text {static }}^{2},
$$

where $\hbar$ is the Planck's constant divided by $2 \pi, \mu$ is the reduced mass for the absorber-scatterer pair, and $\Theta_{E}$ is the Einstein temperature, shown in Fig. 8. $\sigma_{S}^{2}$ is the temperature independent (or static) contribution to the total disorder. The separate thermal $\left(\Theta_{E}\right)$ and structural contributions $\left(\sigma_{S}^{2}\right)$ to the total disorder are listed in Table II.

The vibrational properties of NPs are sensitive to the presence of both capillary pressure and loosely bound, disordered surface atoms that shift the phonon VDOS to higher and lower frequencies, respectively. ${ }^{14,15}$ With reference to Table II, the influence of finite-size effects on the vibrational properties of Ni NPs are readily apparent with a decrease in size leading to a decrease in the $\Theta_{E}$, suggesting that undercoordinated surface atoms are the dominant influence. Similar size-dependent changes in the vibrational properties have been reported for Co (Ref. 16), Cu (Ref. 39), and Sn (Ref. 40) NPs embedded in $\mathrm{SiO}_{2}$ and supported Au NPs. ${ }^{41}$ In contrast, both supported ${ }^{42,43}$ and embedded ${ }^{44} \mathrm{Pt}$ NPs have higher $\Theta_{E}$ relative to bulk material suggesting instead that capillary pressure is the dominant contribution for this metal. Indeed, for a given NP size, the calculated capillary pressure increases as $\mathrm{Au}<\mathrm{Cu}<\mathrm{Ni}<\mathrm{Pt}$ (Ref. 14) and is entirely consistent with the results presented previously.

\section{CONCLUSION}

In summary, $\mathrm{Ni} \mathrm{NPs}$ were formed in $\mathrm{SiO}_{2}$ by ion implantation and thermal annealing. The evolution of the NP size and structure was studied using a combination of SAXS, TEM, XANES, and EXAFS. The average size and distribution of sizes increased with increasing temperature. The calculated activation energy showed that $\mathrm{Ni}, \mathrm{Co}$, and $\mathrm{Cu}$ NPs formed by IBS share common growth kinetics. The NPs were fcc in structure and finite-size effects were readily apparent with the coordination number decreasing and disorder increasing with decreasing NP size. Such trends were consistent with the increasing relative fraction of disordered surface atoms. The bond length also decreased with decreasing NP size as attributed to capillary pressure. An examination of the Ni NP vibrational properties showed that surface effects dominate over those of capillary pressure and induce a shift of the VDOS to lower frequencies manifested by a decrease in Einstein temperature. These results highlight how the NP structural and vibrational properties are intimately linked to finite-size effects and further demonstrate the advantages of IBS for the production of embedded metal NPs.

\section{ACKNOWLEDGMENTS}

This work was financially supported by the Australian Synchrotron and the Australian Research Council with access to equipment provided by the Australian Nanofabrication Facility. Part of this research was undertaken on the SAXS/WAXS beamline at the Australian Synchrotron, Victoria, Australia. D.J.S. thanks J. Bradby and S. Ruffell for helpful suggestions and stimulating scientific discussions.

${ }^{1}$ F. Ercolessi, W. Andreoni, and E. Tosatti, Phys. Rev. Lett. 66, 911 (1991). ${ }^{2}$ S. L. Lai, J. Y. Guo, V. Petrova, G. Ramanath, and L. H. Allen, Phys. Rev. Lett. 77, 99 (1996).

${ }^{3}$ A. Balerna, E. Bernieri, P. Picozzi, A. Reale, S. Santucci, E. Burattini, and S. Mobilio, Phys. Rev. B 31, 5058 (1985).

${ }^{4}$ K. L. Kelly, E. Coronado, L. L. Zhao, and G. C. Schatz, J. Phys. Chem. B 107, 668 (2003).

${ }^{5}$ K. Fukumi, A. Chayahara, K. Kadono, T. Sakaguchi, Y. Horino, M. Miya, K. Fujii, J. Hayakawa, and M. Satou, J. Appl. Phys. 75, 3075 (1994).

${ }^{6}$ I. M. L. Billas, A. Chatelain, and W. A. Deheer, Science 265, 1682 (1994).

${ }^{7}$ A. Meldrum, R. F. Haglund, L. A. Boatner, and C. W. White, Adv. Mater. 13, 1431 (2001).

${ }^{8}$ R. F. Haglund, Mater. Sci. Eng., A 253, 275 (1998).

${ }^{9}$ D. Zanghi, C. M. Teodorescu, F. Petroff, H. Fischer, C. Bellouard, C. Clerc, C. Pelissier, and A. Traverse, J. Appl. Phys. 90, 6367 (2001).

${ }^{10}$ J.-H. Yoon, G.-H. Lee, and R. G. Elliman, J. Appl. Phys. 99, 116106 (2006).

${ }^{11}$ H. Amekura, H. Kitazawa, and N. Kishimoto, Nucl. Instrum. Methods Phys. Res. B 219, 856 (2004).

${ }^{12}$ H. Amekura, N. Umeda, Y. Takeda, J. Lu, and N. Kishimoto, Appl. Phys. Lett. 85, 1015 (2004).

${ }^{13}$ O. Cintora-Gonzalez, C. Estournes, D. Muller, J. Guille, and J. J. Grob, Nucl. Instrum. Methods Phys. Res. B 147, 422 (1999).

${ }^{14}$ R. Meyer, L. J. Lewis, S. Prakash, and P. Entel, Phys. Rev. B 68, 104303 (2003).

${ }^{15}$ A. Kara and T. S. Rahman, Phys. Rev. Lett. 81, 1453 (1998).

${ }^{16}$ P. M. Derlet, S. Van Petegem, and H. Van Swygenhoven, Phys. Rev. B 71, 024114 (2005).

${ }^{17}$ J. Ziegler, J. P. Beirsack, and U. Littmark, The Stopping and Range of Ions in Matter (Pergamon, New York, 1985).

${ }^{18}$ A. Cheung, G. D. Azevedo, C. J. Glover, D. J. Llewellyn, R. G. Elliman, G. J. Foran, and M. C. Ridgway, Appl. Phys. Lett. 84, 278 (2004).

${ }^{19}$ S. R. Kline, J. Appl. Crystallogr. 39, 895 (2006).

${ }^{20}$ B. Ravel and M. Newville, J. Synchrotron Radiat. 12, 537 (2005).

${ }^{21}$ M. Newville, J. Synchrotron Radiat. 8, 322 (2001).

${ }^{22}$ J. J. Rehr and R. C. Albers, Rev. Mod. Phys. 72, 621 (2000).

${ }^{23}$ M. Kotlarchyk and S.-H. Chen, J. Chem. Phys. 79, 2461 (1983).

${ }^{24}$ A. Miotello, G. De Marchi, G. Mattei, P. Mazzoldi, and C. Sada, Phys. Rev. B 63, 075409 (2001).

${ }^{25}$ R. Giulian, L. L. Araujo, P. Kluth, D. J. Sprouster, C. S. Schnohr, B. Johannessen, G. J. Foran, and M. C. Ridgway, J. Appl. Phys. 105, 044303 (2009).

${ }^{26}$ D. J. Sprouster, R. Giulian, L. L. Araujo, P. Kluth, B. Johannessen, D. J. Cookson, G. J. Foran, and M. C. Ridgway, J. Appl. Phys. 107, 014313 (2010).

${ }^{27}$ B. Johannessen, P. Kluth, D. Cookson, G. Foran, and M. Ridgway, Nucl. Instrum. Methods Phys. Res. B 246, 45 (2006).

${ }^{28}$ L. A. Grunes, Phys. Rev. B 27, 2111 (1983).

${ }^{29}$ H. Winnischofer, T. C. R. Rocha, W. C. Nunes, L. M. Socolovsky, M. Knobel, and D. Zanchet, ACS Nano 2, 1313 (2008).

${ }^{30}$ B. Johannessen, P. Kluth, C. J. Glover, G. D. Azevedo, D. J. Llewellyn, G. J. Foran, and M. C. Ridgway, J. Appl. Phys. 98, 024307 (2005).

${ }^{31}$ P. Kluth, B. Johannessen, V. Giraud, A. Cheung, C. J. Glover, G. D. Azevedo, G. J. Foran, and M. C. Ridgway, Appl. Phys. Lett. 85, 3561 (2004).

${ }^{32}$ L. L. Araujo, R. Giulian, D. J. Sprouster, C. S. Schnohr, D. J. Llewellyn, P. Kluth, D. J. Cookson, G. J. Foran, and M. C. Ridgway, Phys. Rev. B 78, 094112 (2008).

${ }^{33}$ C. W. Mays, J. S. Vermaak, and D. Kuhlmann-Wilsdorf, Surf. Sci. 12, 134 (1968).

${ }^{34}$ H. P. Wang, J. Chang, and B. Wei, J. Appl. Phys. 106, 033506 (2009).

${ }^{35}$ G. Apai, J. F. Hamilton, J. Stohr, and A. Thompson, Phys. Rev. Lett. 43, 165 (1979).

${ }^{36}$ D. J. Sprouster, R. Giulian, L. L. Araujo, P. Kluth, B. Johannessen, N. Kirby, K. Nordlund, and M. C. Ridgway, Phys. Rev. B 81, 155414 (2010). 
${ }^{37}$ L. L. Araujo, P. Kluth, G. d. M. Azevedo, and M. C. Ridgway, Phys. Rev. B 74, 184102 (2006).

${ }^{38}$ A. Frenkel and J. Rehr, Phys. Rev. B 48, 585 (1993).

${ }^{39}$ P. Kluth, B. Johannessen, L. L. Araujo, and M. C. Ridgway, AIP Conf. Proc. 882, 731 (2007).

${ }^{40}$ G. E. J. Koops, H. Pattyn, A. Vantomme, S. Nauwelaerts, and R. Venegas, Phys. Rev. B 70, 235410 (2004).
${ }^{41}$ T. Comaschi, A. Balerna, and S. Mobilio, Phys. Rev. B 77, 075432 (2008).

${ }^{42}$ J. H. Kang, L. D. Menard, R. G. Nuzzo, and A. I. Frenkel, J. Am. Chem. Soc. 128, 12068 (2006).

${ }^{43}$ B. Roldan Cuenya, A. I. Frenkel, S. Mostafa, F. Behafarid, J. R. Croy, L. K. Ono, and Q. Wang, Phys. Rev. B 82, 155450 (2010).

${ }^{44}$ R. Giulian, L. L. Araujo, P. Kluth, D. J. Sprouster, C. S. Schnohr, G. J. Foran, and M. C. Ridgway, J. Phys.: Condens. Matter 21, 155302 (2009). 